\section{Identification of phosphatases for Smad in the BMP/DPP pathway}

\author{
Hong B. Chen, Jiali Shen, Y. Tony Ip, and \\ Lan $\mathrm{Xu}^{1}$
}

Program in Molecular Medicine, University of Massachusetts Medical School, Worcester, Massachusetts 01605, USA

\begin{abstract}
Phosphorylation of the SSXS motif of Smads is critical in activating the transforming growth factor $\beta$ (TGF- $\beta$ ) and bone morphogenetic protein (BMP) pathways. However, the phosphatase(s) involved in dephosphorylating and hence inactivating Smads remained elusive. Through RNA interference (RNAi)-based screening of serine/ threonine phosphatases in Drosophila S2 cells, we identified pyruvate dehydrogenase phosphatase (PDP) to be required for dephosphorylation of Mothers against Decapentaplegic (MAD), a Drosophila Smad. Biochemical and genetic evidence suggest that PDP directly dephosphorylates MAD and inhibits signal transduction of Decapentaplegic (DPP). We show that the mammalian PDPs are important in dephosphorylation of BMP-activated Smad1 but not TGF- $\beta$-activated Smad2 or Smad3. Thus, PDPs specifically inactivate Smads in the BMP/ DPP pathway.
\end{abstract}

Received October 14, 2005; revised version accepted January 13, 2006.

The transforming growth factor $\beta$ (TGF- $\beta$ ) cytokines share an evolutionarily conserved mechanism of signal transduction (Attisano and Wrana 2002; Shi and Massagué 2003; ten Dijke and Hill 2004). Genetic analyses of the DPP (Decapentaplegic) pathway in Drosophila identified MAD (Mothers against Decapentaplegic) as a key signal transducer (Raftery et al. 1995; Sekelsky et al. 1995). The orthologs of MAD in mammals include Smad 2 and Smad3 in the TGF- $\beta$ pathway, and Smad1, Smad5, and Smad8 in the bone morphogenetic protein (BMP) pathway (Derynck et al. 1998; Whitman 1998). These Smads (referred to as R-Smads) contain an SSXS motif at their $C$ termini, and the last two serines are phosphorylated in response to TGF- $\beta$ or BMP (MaciasSilva et al. 1996; Kretzschmar et al. 1997; Souchelnytskyi et al. 1997). This phosphorylation is a prerequisite for the Smads to accumulate in the nucleus and to interact with transcription cofactors (Chen et al. 1996; Lagna et al. 1996; Abdollah et al. 1997).

The C-terminal phosphorylation state of R-Smads is controlled by protein kinases and phosphatases. When the TGF- $\beta$ receptor kinase activity was blocked, the

[Keywords: Bone morphogenetic protein; Decapentaplegic; Mothers against Decapentaplegic; Smad; pyruvate dehydrogenase phosphatase] ${ }^{1}$ Corresponding author.

E-MAIL lan.xu@umassmed.edu; FAX (508) 856-6662.

Article published online ahead of print. Article and publication date are at http://www.genesdev.org/cgi/doi/10.1101/gad.1384706. amount of phospho-Smad2/3 rapidly decreased with concomitant return of Smad2/3 to the cytoplasm (Inman et al. 2002; Xu et al. 2002). While degradation may account for part of the loss of phospho-Smad2/3, dephosphorylation plays a major role in inactivating R-Smads (Lo and Massagué 1999; Inman et al. 2002). However, the identity of Ser/Thr phosphatase(s) involved in dephosphorylation of R-Smads was unknown.

In this study, we uncovered pyruvate dehydrogenase phosphatase (PDP) to be a phosphatase for MAD. We found that genetic lesions in the $p d p$ gene resulted in ectopic accumulation of C-terminally phosphorylated MAD in Drosophila embryos. Furthermore, we demonstrate that PDP directly dephosphorylates MAD and inhibits DPP-induced gene expression. Moreover, knockdown of mammalian orthologs of PDP (PDP1 and PDP2) by small interfering RNA (siRNA) led to reduced dephosphorylation of Smad1, but not Smad2 or Smad3, suggesting that PDPs specifically regulate BMP signaling in mammalian cells.

\section{Results and Discussion \\ Identification of a Ser/Thr phosphatase required for dephosphorylation of DPP-activated MAD}

In Drosophila S2 cells, the level of DPP-induced phosphorylation of MAD remained elevated in the continuous presence of DPP (Fig. 1A). However, upon removal of DPP, the amount of phospho-MAD decreased rapidly while the total protein level of MAD was unchanged (Fig. 1B). Thus, we reason that phospho-MAD is dephosphorylated once the DPP signal subsides.

To identify phosphatase(s) for MAD, we screened a previously published library of double-stranded RNAs (dsRNAs) against all $44 \mathrm{Ser} / \mathrm{Thr}$ phosphatases in the Drosophila genome using RNA interference (RNAi) (Clemens et al. 2000; Lum et al. 2003). The dsRNA against CG12151 (CG12151-A, targeting the Drosophila PDP) consistently impeded dephosphorylation of MAD (Fig. 1C,D). A similar observation was made with a second dsRNA for PDP (CG12151-B) targeting a different region (Fig. 1C,D). We noticed that eventually (i.e., $2 \mathrm{~h}$ after removal of DPP), the level of phospho-MAD decreased substantially even when PDP was knocked down (Fig. 1C,D). This could reflect activities from residual PDP or other phosphatases that can compensate partially. Another possibility is that if phospho-MAD constitutes a very small fraction of the total MAD, then degradation of phospho-MAD specifically may also result in reduction of phospho-MAD without grossly changing the level of total MAD.

In parallel to the decrease in phospho-MAD, the expression of Daughters against Decapentaplegic (DAD), a DPP-dependent target gene, dropped substantially from the peak DPP-induced level $2 \mathrm{~h}$ after DPP removal (Fig. 1E). dsRNA against PDP significantly reduced such decrease in $D A D$ expression, consistent with its ability to delay dephosphorylation of MAD (Fig. 1E).

\section{Loss of PDP results in ectopic phosphorylation of $M A D$ in Drosophila embryos}

To further determine the importance of PDP in regulating the C-terminal phosphorylation state of MAD, we 
A

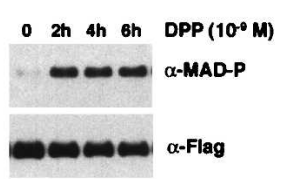

B

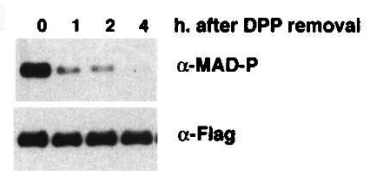

C

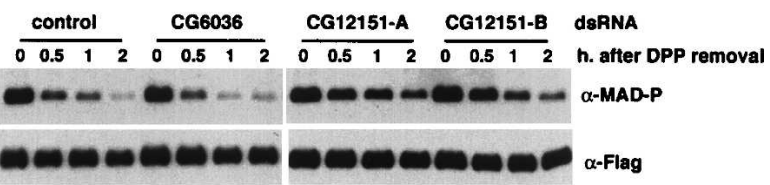

D

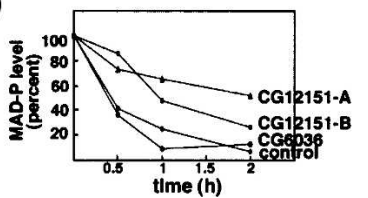

E

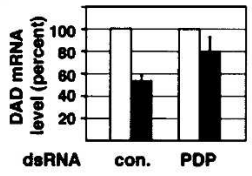

Figure 1. Identification of PDP as a phosphatase required for dephosphorylation of MAD. $(A)$ Continuous presence of DPP resulted in persistent phosphorylation of MAD for up to $6 \mathrm{~h}$. Flag-MAD was inducibly expressed in S2 cells followed by DPP treatment. C-terminally phosphorylated MAD (having the same SSXS and adjacent residues as Smad1) was detected by anti-phospho-Smad1 ( $\alpha$-MADP). (B) The same S2 cells as in $A$ were first stimulated by DPP for 2 $\mathrm{h}$ and washed by PBS to remove DPP, and continued to be cultured in regular media. At indicated time points, phospho-MAD and total Flag-MAD were detected by the indicated antibodies. $(C)$ The same S2 cell line was treated with the indicated dsRNAs for $72 \mathrm{~h}$ before being analyzed as in $B$. (D) Quantitation of phospho-MAD signal in $C$. The signal of phospho-MAD was quantitated using NIH Image, normalized to that of total Flag-MAD, and plotted. The phosphoMAD level at the 0 time point was arbitrarily set as $100 \%$. $(E)$ The $D A D$ mRNA level was measured by quantitative real-time PCR at the peak DPP-induced level $\left(10^{-9} \mathrm{M}\right.$ for $2 \mathrm{~h}$ ) (white bars) and $2 \mathrm{~h}$ after DPP removal (black bars). The peak level was arbitrarily set as $100 \%$. The mRNA level of the ribosomal protein $r p 49$ was used as the quantitation standard. The presented values are based on four independent experiments.

examined Drosophila strains carrying genetic lesions that affect the PDP locus on the $\mathrm{X}$ chromosome (division 7B7). The strain PBacRBCG12151 e2351 $^{\text {(PBac }}{ }^{\text {CG12151) }}$ harbors a piggyback transposon 7 base pairs upstream of the first exon of the PDP gene (Thibault et al. 2004). Another strain, $D f(1) c t 4 b 1$ (uncovers 7B2-7C4), carries a chromosome deletion that includes the PDP gene. We stained whole embryos with the PS1 antisera that specifically recognize phospho-MAD in Drosophila embryos (Persson et al. 1998; Tanimoto et al. 2000; Sutherland et al. 2003).

Similar to previous reports, in wild-type blastodermstage embryos, phospho-MAD is distributed only in the dorsal-most cells and predominantly nuclear (Fig. 2A,B; Sutherland et al. 2003). In the PBac ${ }^{\text {CG12151 }}$ and $D f(1) c t 4 b 1$ embryos, in addition to the dorsal nuclei, we observed punctate staining for phospho-MAD throughout the embryo (Fig. 2A,C-E). The ectopic PS1 staining was mostly outside the nucleus (Fig. 2C-E), and was detected in embryos at most, if not all, developmental stages (Fig. 2A). The ectopic accumulation of phosphoMAD was not observed in the Df(1)RF19 (uncovers 7A47B3) strain, with a chromosome deletion adjacent to but not encompassing the PDP locus (data not shown). In control experiments, no abnormal staining patterns for Dorsal (data not shown) and Toll were observed in either $\mathrm{PBac}^{\mathrm{CG} 12151}$ or $\mathrm{Df}(1)$ ct4b1 embryos (Fig. 2A). In cleav-

age-stage embryos, which undergo rapid mitosis, we detected aggregated signals for phospho-MAD in $\mathrm{PBac}^{\mathrm{CG} 12151}$ and $D f(1) c t 4 b 1$, but no PS1 signal in wildtype embryos (Fig. 2A,E). Double staining with DAPI showed that in these mutant embryos, phospho-MAD accumulated on both sides of the condensed chromosomes (Fig. 2E).

Genetic analysis showed that the ectopic phosphorylation of MAD is a maternal effect phenotype. All embryos from crosses between heterozygous mutant females and wild-type males displayed phenotypes as shown in Figure 2. If heterozygous males were crossed with wild-type females, no embryos showed any ectopic PS1 staining (data not shown). Therefore, haploinsufficiency of PDP has a pronounced maternal effect on the level and distribution of phospho-MAD in the early embryos, suggesting that PDP is a critical regulator of the C-terminal phosphorylation state of MAD in vivo.

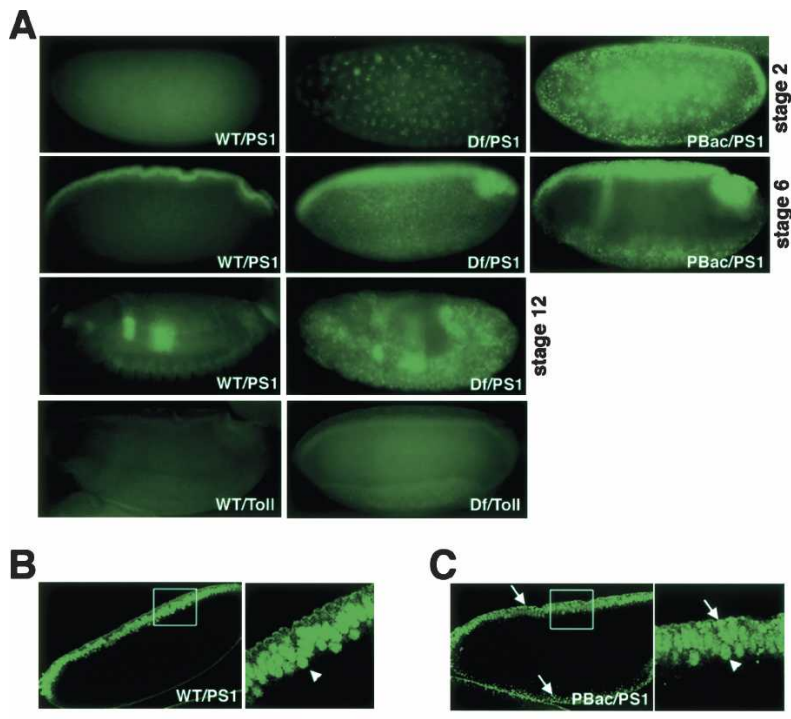

D

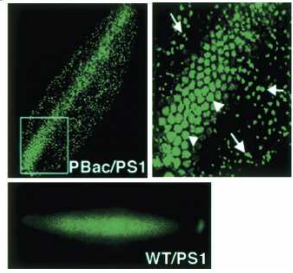

E

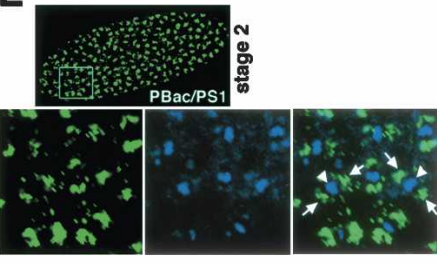

Figure 2. Genetic aberrations of $p d p$ lead to ectopic accumulation of phospho-MAD in Drosophila embryos. (A) Immunostaining of Drosophila embryos using the PS1 antisera. Side views of the wildtype (WT), $D f(1) c t 4 b 1$ (Df), and PBac ${ }^{\mathrm{CG} 12151}$ (PBac) embryos at indicated developmental stages. Note the punctate PS1 staining in mutant embryos. As a control, no punctate pattern was observed for Toll. $(B, C)$ PS1 staining of wild-type $(B)$ and PBac ${ }^{C G 12151}(C)$ stage 6 embryos (side views). The magnified boxed areas are shown on the right. The arrowheads point to phospho-MAD in the nuclei of dorsal midline cells, and the arrows point to the ectopic punctate staining of phospho-MAD observed only in the mutant. (D) PS1 staining of stage 6 wild-type (bottom) and PBac ${ }^{\mathrm{CG} 12151}$ embryos from the dorsal view. The magnified boxed area is shown on the top right. The arrowheads and arrows are as described in $C$. $(E)$ Phospho-MAD staining of PBac ${ }^{\mathrm{CG} 12151}$ embryos at stage 2. Magnified versions of the boxed area are shown in the bottom panels, including the overlay of PS1 (green) and DAPI (blue) staining. The arrowheads mark the chromosomes, and the arrows point to phospho-MAD. 


\section{PDP directly dephosphorylates DPP-activated MAD}

Thickveins (TKV) and Punt are the receptor kinases upstream of MAD that are themselves activated by phosphorylation (Raftery and Sutherland 1999). Conceivably, phosphatases toward either TKV or Punt could also impact the level of phospho-MAD. We therefore determined if PDP directly dephosphorylates MAD as its substrate.

Recombinant GST-PDP effectively dephosphorylated phospho-MAD (Fig. 3A), at as low as $0.3 \mu \mathrm{M}$ (Fig. 3B). Removal of the GST moiety by thrombin did not affect the efficiency of dephosphorylation (Fig. 3A). Asp93 in PDP is highly conserved and critical for metal ion chelating (Das et al. 1996; Kusuda et al. 1998). When Asp93 was mutated to Ala, the phosphatase activity toward MAD was largely abolished (Fig. 3A,B). As controls for specificity in the in vitro phosphatase assay, PDP was inactive against phospho-Jun kinase 1 (JNK1 phosphory-
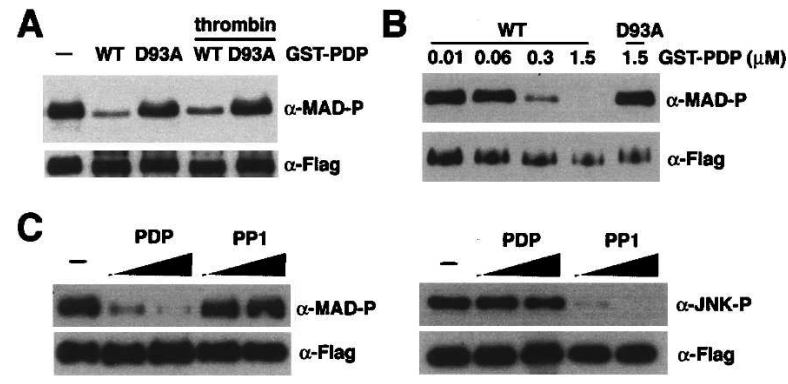

D

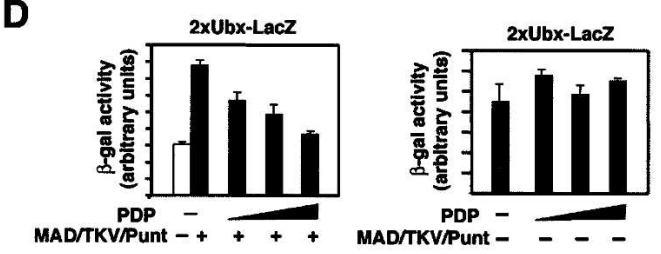

E

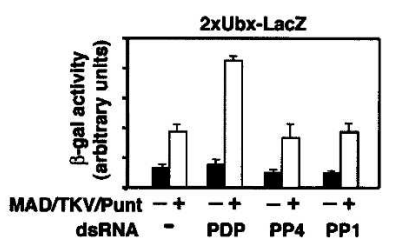

Figure 3. PDP directly dephosphorylates MAD and inhibits DPPresponsive reporter expression. (A) In vitro phosphatase assay. Purified GST-PDP or the D93A mutant was used as the enzyme, with or without GST removal by thrombin as indicated. Flag-MAD expressed in DPP $\left(10^{-9} \mathrm{M}\right)$-treated S2 cells was immunoprecipitated and used as the substrate. Phospho-MAD and total Flag-MAD were detected by anti-phospho-Smad1 and anti-Flag immunoblotting, respectively. (B) Phosphatase assay as in $A$ using different concentrations of GST-PDP. (C) In vitro phosphatase assay using phosphoMAD (left) or phospho-JNK1 (right) as the substrate. As indicated, recombinant GST-PDP $(0.3 \mu \mathrm{M}, 1.5 \mu \mathrm{M})$ or PP1 (2 U, $10 \mathrm{U})$ was used. Phospho- and total Flag-JNK1 were detected by anti-phospho-JNK1 and anti-Flag immunoblotting, respectively. $(D$, left $)$ MAD-activated $2 \mathrm{x} U b \mathrm{x}-\mathrm{lacZ}$ expression in S2R + cells was repressed by PDP in a dose-dependent manner. (Right) The same PDP overexpression did not affect the basal reporter expression in the absence of activated MAD. A Tk-luc reporter was cotransfected as an internal control, and the presented value of $\beta$-gal activity has been normalized to the luciferase activity. (E) S2R+ cells treated with indicated dsRNAs (PP4: CG11597; PP1: CG8822) were subjected to reporter assay as in $D$. lated on Thr185) under the same conditions (Fig. 3C, right); and phospho-MAD was not dephosphorylated by protein phosphatase 1 (PP1), which exhibited strong activity toward phospho-JNK1 (Fig. 3C).

Therefore, C-terminally phosphorylated MAD is a bona fide substrate of PDP. In eukaryotes, Ser/Thr phosphatases are grouped into PPP and PPM families (Barford et al. 1998). PDPs belong to the PPM family whose catalytic domains are similar to that of PP2C, and pyruvate dehydrogenase was the only known substrate of PDPs (Lawson et al. 1993; Simonot et al. 1997; Huang et al. 1998). We have thus identified a novel substrate and function for PDP.

\section{PDP inhibits DPP signaling}

The C-terminal phosphorylation is critical for MAD to regulate gene transcription. We therefore tested if PDP could inhibit MAD-mediated transcriptional activation. In the Drosophila S2R+ cells, 2xUbx-lacZ, a reporter controlled by a DPP response element from the Ultrabithorax promoter, was activated upon expression of MAD, Medea (MED), Punt, and TKV, as previously reported (Fig. 3D; Kirkpatrick et al. 2001). The expression of this reporter was significantly repressed when PDP was overexpressed in a dose-dependent manner (Fig. 3D). Moreover, dsRNA against PDP, but not dsRNAs against PP1-like (CG8822) or PP4-like (CG11597) Ser/Thr phosphatases, enhanced the expression of $2 \mathrm{x} U b x$-lacZ upon activation of MAD (Fig. 3E). Either overexpression or knockdown of PDP had little effect on the basal $2 x U b x-$ lacZ expression (Fig. 3D [right], E). Thus, the level of PDP is an important determinant of the strength of DPP signaling.

\section{Interaction between PDP and MAD}

After DPP stimulation, endogenous phospho-MAD was readily coimmunoprecipitated with V5-tagged PDP (Fig. 4A). In S2 cells transfected with Flag-MAD and PDP-V5, anti-Flag immunoprecipitation brought down PDP-V5, demonstrating that the coimmunoprecipitation of MAD and PDP works in reciprocal order (Fig. 4B). Anti-V5 immunoblotting revealed two V5-containing proteins in cells transfected with PDP-V5, suggesting the presence of different forms of PDP (input in Fig. 4A,B).

We further investigated the contribution of C-terminal phosphorylation of MAD in its interaction with PDP. Phospho-MAD was bound equally well by wild-type and the D93A mutant form of GST-PDP (Fig. 4C). Unphosphorylated MAD interacted with PDP (Fig. 4D, right panel). However, when compared with the input, $\sim 3 \%$ of phospho-MAD was bound by GST-PDP; while $<1 \%$ of unphosphorylated MAD was bound (Fig. 4D). Thus, while not being required for interaction, the C-terminal phosphorylation substantially enhanced MAD interaction with PDP.

\section{Subcellular localization of PDP}

In live S2 cells, PDP-GFP not only distributed throughout the cell, but also exhibited a punctate pattern that overlapped with mitochondria as revealed by MitoTracker (Fig. 4E). Such distribution of PDP was not changed upon activation of the DPP pathway (Fig. 4F). Overexpression of PDP-GFP did not apparently prevent 
A

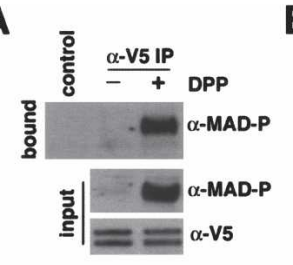

D

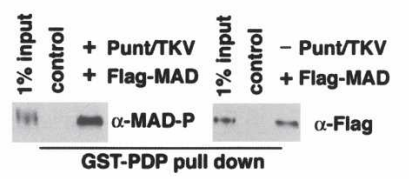

$\mathbf{F}$

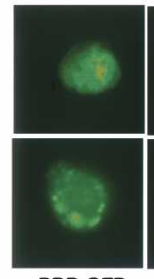

PDP.GFP

B

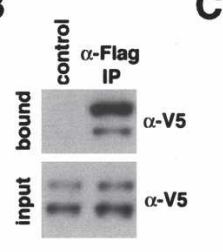

E

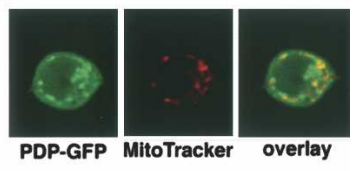

G

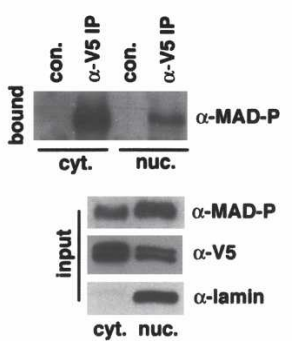

Figure 4. PDP interaction with MAD and its subcellular localization. (A) Coimmunoprecipitation of endogenous phospho-MAD and PDP-V5. S2 cells were induced to express PDP-V5 followed by DPP $\left(10^{-9} \mathrm{M}\right)$ treatment. The whole-cell extract was immunoprecipitated by anti-V5, and the bound proteins were analyzed by anti-phosphoSmadl. Protein A + G beads alone were used as the control. Expression of PDP-V5 and endogenous phospho-MAD was detected by indicated antibodies (input). (B) S2 cells expressing Flag-MAD and PDP-V5 were stimulated by DPP $\left(10^{-9} \mathrm{M}\right)$, and the whole-cell extract was subject to immunoprecipitation with anti-Flag. The bound and input proteins were immunoblotted with anti-V5. (C) S2 cells coexpressing Flag-MAD, Punt, and TKV were used to prepare cell extract containing phospho-MAD and used in GST pull-down using wild-type GST-PDP or the D93A mutant. Bound proteins were analyzed by anti-phospho-Smadl immunoblotting. GST beads alone were used as the control. (D) GST-pull-down experiments comparing PDP interaction with phosphorylated or unphosphorylated MAD. Extracts from S2 cells expressing Flag-MAD with or without Punt and TKV were used as the source of phospho- or unphosphorylated MAD, respectively. $(E)$ Live S2 cells expressing PDP-GFP were stained with MitoTracker Deep Red 633, and the fluorescent images were captured by confocal microscopy. $(F)$ S2 cells expressing PDPGFP and Flag-MAD were stained with anti-Flag and analyzed by fluorescent microscopy. Punt and TKV were coexpressed as indicated to induce nuclear accumulation of Flag-MAD. $(G)$ Coimmunoprecipitation of PDP and phospho-MAD in cytosol and nucleus. S2 cells transfected with PDP-V5 and Flag-MAD were stimulated with DPP before nuclear and cytosolic fractions were prepared for immunoprecipitation with anti-V5. The bound proteins were analyzed by anti-phospho-Smad1. Lamin was detected exclusively in the nuclear fraction, validating the subcellular fractionation procedure.

nuclear accumulation of MAD, possibly because the receptor activation overrode PDP activity under the condition or because changes in the kinetics of MAD nuclear import/export require more sensitive and quantitative methods to measure (Fig. 4F). Our observations suggest that PDP has broad subcellular localization and can therefore gain access to substrates in mitochondria, cytoplasm, and nucleus. Indeed, in both the cytosolic and nuclear fractions, coimmunoprecipitation of phosphoMAD and PDP could be detected (Fig. 4G). Less phosphoMAD was coimmunoprecipitated with PDP in the

nuclear fraction, which could be due to the fact that less PDP was present in the nucleus and that the buffer used to extract nuclei contained a higher salt and detergent concentration, which is more stringent for protein-protein interaction (Fig. 4G).

\section{Mammalian PDPs are involved in dephosphorylation of BMP-activated Smad1}

We next investigated if mammalian PDPs had similar functions to their Drosophila counterpart. Two orthologs of Drosophila PDP, PDP1 and PDP2, have been identified in the human genome, and both share $\sim 40 \%$ identity with the Drosophila PDP in amino acid sequences (Lawson et al. 1993; Simonot et al. 1997; Huang et al. 1998). Coimmunoprecipitation between PDP2 and Smad1 was detected in $293 \mathrm{~T}$ cells, suggesting that the functional interaction between PDP and Smad is conserved in mammalian cells (Fig. 5A).

To further test this, we designed two siRNA duplexes for each of PDP1 and PDP2 (siPDP1-a,b and siPDP2-a,b), which correspondingly reduced the mRNA levels of PDP1 and PDP2 (Fig. 5B). The TGF- $\beta$ receptor kinases can be inhibited by the compound SB431542 (Inman et al. 2002), while SB202190 was shown to block BMP re-

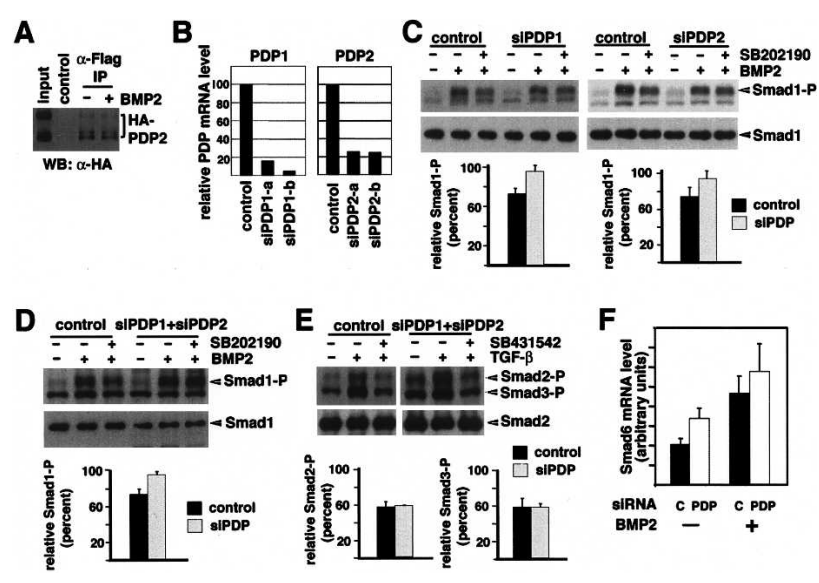

Figure 5. Knockdown of PDP1 and PDP2 affect dephosphorylation of Smad1 in mammalian cells. (A) 293T cells expressing Flag-Smad1 and HA-PDP2 were treated with BMP2 as indicated before wholecell extract was prepared for anti-Flag immunoprecipitation. The bound proteins were analyzed by anti-HA. $(B)$ HeLa cells were transfected with indicated siRNA duplexes, and the mRNA levels of PDP1 and PDP2 were measured $72 \mathrm{~h}$ later by quantitative real-time RT-PCR. $(C)$ siRNAs against PDPs inhibited dephosphorylation of Smad1. HeLa cells transfected with control, siPDP1 (siPDP1-a and 1-b), or siPDP2 (siPDP2-a and 2-b) siRNA duplexes were analyzed for dephosphorylation of Smadl as described in Materials and Methods. (Bottom) The reduction in phospho-Smad1 level after SB202190 treatment was quantitated as the percentage of the phospho-Smadl level before SB202190 treatment. NIH Image was used to quantitate immunoblot signals from three separate experiments, and the plotted values have been normalized to the total Smadl level. $(D, E)$ HeLa cells transfected with control or combined siPDP1-a and siPDP2-a siRNA duplexes were analyzed for dephosphorylation of Smad1 $(D)$ and Smad2/3 $(E)$. The quantitation was done as in $C$, with the level of total Smad1 $(D)$ or Smad2 $(E)$ serving as the normalization standard. (F) Combined siRNA against PDPs (PDP), but not the control siRNA $(\mathrm{C})$, enhanced expression of Smad6 in HeLa cells. Quantitative real-time PCR was used to measure Smad6 mRNA level before and after BMP2 treatment (100 ng/mL, $2 \mathrm{~h}$ ), using $18 \mathrm{~s}$ rRNA as the quantitation standard. The plotted values are based on three independent experiments. 
ceptor kinase activity toward Smad1 (Nishihara et al. 2003). In HeLa cells, TGF- $\beta$ - and BMP2-induced phosphorylation of Smad2/3 and Smad1 decreased considerably after SB431542 or SB202190 treatment, respectively, reflecting dephosphorylation of these Smads (Fig. 5C-E). Therefore, we used these two kinase inhibitors to monitor phosphatase activities toward Smad1, Smad2, and Smad3.

Compared with the control, siRNA against PDP1 or PDP2 resulted in reduced Smad1 dephosphorylation (Fig. 5C). However, the same siRNA treatment did not significantly change the dephosphorylation of Smad2 and Smad3 (data not shown; but see Fig. 5E). Even when we combined siRNAs against PDP1 and PDP2, dephosphorylation of Smad2 and Smad3 was largely unaffected (Fig. 5D,E). Moreover, the expression of one BMP target gene, Smad6, was enhanced in cells transfected with siRNA against PDP1 and PDP2, both at the basal state and after BMP2 stimulation (Fig. 5F). The increase in Smad6 expression without added BMP2 could reflect enhancement of low-level autocrine BMP signaling.

These observations suggest that PDP1 and PDP2 are important for dephosphorylation of Smad1. Therefore, the mechanism of Smad dephosphorylation in vertebrates is similar to that in Drosophila. BMP is more closely related to DPP, and Smad1 is more similar to MAD than Smad2/3 are. Interestingly PDP1 and PDP2 did not appear to be rate-limiting in dephosphorylation of Smad2 and Smad3. This raised the possibility that different phosphatases are used to inactivate different R-Smads.

The activation state of various signal transduction pathways is often dictated by phosphorylation-dephosphorylation control of key signaling molecules. We report here that PDPs are phosphatases for R-Smads in the DPP pathway in Drosophila and the BMP pathways in mammals. PDP can act to reduce the concentration of phospho-R-Smads in the nucleus and consequently weaken the transcriptional responses to BMP. Moreover, our findings also raised the possibility that MAD may be phosphorylated by kinases outside of the DPP pathway, and the role of PDP is to remove such aberrant phosphorylation and prevent ectopic DPP signaling.

TGF- $\beta$ /BMP cytokines control the biology of many cell types in different physiological contexts (Massagué et al. 2000). In addition to the receptors and Smads, other factors must participate to modify and fine-tune the signaling. The identification of phosphatases that inactivate R-Smads provides us with a new angle to study how TGF- $\beta /$ BMP signaling is modulated.

\section{Materials and methods}

Cell culture, transfection, immunocytochemistry, and reporter assay Drosophila S2 and S2R+ cells were cultured at $26^{\circ} \mathrm{C}$ in Schneider's medium (Invitrogen) with $10 \%$ fetal bovine serum and $100 \mathrm{U} / \mathrm{mL}$ penicillin/ streptomycin. Cells were transfected using Effectene (Qiagen). For mitochondria staining, cells were cultured with $100 \mathrm{nM}$ MitroTracker Deep Red 633 (Invitrogen) for $1 \mathrm{~h}$, washed twice, and then observed directly using confocal microscopy (Leica). Drosophila embryo fixation and immunostaining were carried out as previously described (Hemavathy et al. 2004). The PS1 antisera for phospho-MAD (1:1000) were a gift from Dr. P. ten Dijke (The Netherlands Cancer Institute, Amsterdam, The Netherlands).

In reporter assays, transfected cells were induced with $0.5 \mathrm{mM} \mathrm{CuSO}_{4}$ to express MAD, PDP, Punt, and TKV as indicated. A TK promoterdriven luciferase reporter was used as a normalization standard. Cell lysates were assayed for both luciferase and $\beta$-galactosidase activities (Promega).
Cell-based assay for phosphatase activity against MAD and Smad A stable S2 cell line was generated expressing Flag-MAD under the control of the metallothionine promoter. The cells were induced with 0.5 $\mathrm{mM} \mathrm{CuSO}_{4}$ overnight and then treated with $10^{-9} \mathrm{M}$ DPP (R\&D Systems) for $2 \mathrm{~h}$. After three washes with PBS, the cells were cultured in the absence of DPP. At indicated time points, cells were harvested and analyzed by immunoblotting using anti-phospho-Smadl (Cell Signaling).

HeLa cells were starved in 1\% FBS/DMEM media for $3 \mathrm{~h}$ before being treated with $100 \mathrm{ng} / \mathrm{mL}$ BMP2 (R\&D Systems) or $100 \mathrm{pM}$ TGF- $\beta$ (R\&D Systems) for $1 \mathrm{~h}$. The cells were then treated with $10 \mu \mathrm{M}$ SB431542 (Tocris) or $20 \mu \mathrm{M}$ SB202190 (Calbiochem) for $30 \mathrm{~min}$ before being harvested and analyzed by immunoblotting.

RNAi and quantitative real-time PCR

For RNAi in Drosophila cells, PCR amplicons of targeted cDNA were transcribed in vitro using the MEGAscript kit (Ambion) to generate dsRNAs, which were introduced into S2 cells as described previously (Clemens et al. 2000). siRNA duplexes against PDP1 and PDP2 (SiPDP1a, CAAGTTGGTGATCCTAATT; siPDP1-b, CAGTCACGCTGTCTA ATGA; siPDP2-a, GAAGCATTAATGTACTCCT; and siPDP2-b, CA ATCGGTGCATATTACAA) were provided by Dr. Biliang Zhang (Ribobio Co. Ltd, Guangzhou, China). siRNAs (25-40 nM final) were transfected using HiperFect (Qiagen). After 72 h, the transfection was repeated, and another $48 \mathrm{~h}$ later, cells were harvested for analyses.

Real-time PCR was carried out using the iQ SYBR Green Supermix (Bio-Rad). Total RNA was extracted using the RNeasy kit (Qiagen), and cDNA was synthesized with the iScript cDNA Synthesis Kit (Bio-Rad). The level of test cDNA relative to that of 18s rRNA or ribosomal protein rp49 was calculated by the $2^{-\Delta \Delta \mathrm{CT}}$ method (Livak and Schmittgen 2001).

In vitro phosphatase assay

S2 cells were transfected with Flag-MAD and treated with $10^{-9} \mathrm{M}$ DPP for $2 \mathrm{~h}$ to generate phospho-MAD. 293T cells transfected with Flag-JNK1 were exposed to UV light $\left(60 \mathrm{~J} / \mathrm{m}^{2}\right)$ to induce phosphorylation of JNK1. Cells were lysed in $20 \mathrm{mM}$ Tris- $\mathrm{Cl}$ (pH 7.4), $150 \mathrm{mM} \mathrm{NaCl}, 0.5 \%$ NP-40, and Flag-MAD or Flag-JNK1 was immunoprecipitated with anti-Flag (Sigma). After washing in the lysis buffer, the beads were suspended in 50 $\mathrm{mM}$ Tris-Cl (pH 7.4) and $5 \mathrm{mM} \mathrm{MnCl}_{2}$, and mixed with GST-PDP or PP1 (Calbiochem). The reaction was carried out at $30^{\circ} \mathrm{C}$ for $30 \mathrm{~min}$.

Protein-protein interaction

For GST pull-down, cell lysate was prepared by sonication in $20 \mathrm{mM}$ Tris (pH 7.4), $175 \mathrm{mM} \mathrm{NaCl}, 0.5 \%$ Triton X-100, and $2 \mathrm{mM}$ DTT with protease inhibitors. Such a whole-cell extract was incubated with GST-PDP on ice for $1 \mathrm{~h}$ before adding glutathione-conjugated agarose beads. After $1 \mathrm{~h}$ at $4^{\circ} \mathrm{C}$, the beads were washed three times in the lysis buffer and analyzed by immunoblotting. For coimmunoprecipitation, cells were lysed in $20 \mathrm{mM}$ Tris-Cl (pH 7.4), $150 \mathrm{mM} \mathrm{NaCl}, 0.5 \% \mathrm{NP}-40$, and $20 \mathrm{mM}$ DTT and immunoprecipitated with anti-Flag (Sigma) or anti-V5 agarose beads (Invitrogen) for $5 \mathrm{~h}$ at $4^{\circ} \mathrm{C}$. The beads were washed three times in the lysis buffer.

Subcellular fractionation was done using the NE-PER nuclear and cytoplasmic extraction reagents following the manufacturer's protocol (Pierce).

\section{Acknowledgments}

We are grateful to Drs. P. ten Dijke, R. Padgett, L. Lum, P. Beachy, R. Davis, and A. Laughon for valuable reagents, and Dr. B. Zhang for siRNA duplexes. We thank Drs. R. Davis and Q. Xu for suggestions and critical reading of the manuscript. This work is supported by a grant from the National Institutes of Health to L.X.

\section{References}

Abdollah, S., Macias-Silva, M., Tsukazaki, T., Hayashi, H., Attisano, L. and Wrana, J.L. 1997. T $\beta$ RI phosphorylation of Smad2 on Ser465 and Ser467 is required for Smad2-Smad4 complex formation and signaling. J. Biol. Chem. 272: 27678-27685.

Attisano, L. and Wrana, J.L. 2002. Signal transduction by the TGF- $\beta$ superfamily. Science 296: 1646-1647.

Barford, D., Das, A.K., and Egloff, M.P. 1998. The structure and mecha- 
nism of protein phosphatases: Insights into catalysis and regulation Annu. Rev. Biophys. Biomol. Struct. 27: 133-164.

Chen, X., Rubock, M.J., and Whitman, M. 1996. A transcriptional partner of MAD proteins in TGF- $\beta$ signalling. Nature 383: 691-696.

Clemens, J.C., Worby, C.A., Simonson-Leff, N., Muda, M., Maehama, T., Hemmings, B.A., and Dixon, J.E. 2000. Use of double-stranded RNA interference in Drosophila cell lines to dissect signal transduction pathways. Proc. Nat1. Acad. Sci. 97: 6499-6503.

Das, A.K., Helps, N.R., Cohen, P.T., and Barford, D. 1996. Crystal structure of the protein serine/threonine phosphatase $2 \mathrm{C}$ at $2.0 \AA$ resolution. EMBO J. 15: 6798-6809.

Derynck, R., Zhang, Y., and Feng, X.H. 1998. Smads: Transcriptional activators of TGF- $\beta$ responses. Cell 95: 737-740.

Hemavathy, K., Hu, X., Ashraf, S.I., Small, S.J., and Ip, Y.T. 2004. The repressor function of snail is required for Drosophila gastrulation and is not replaceable by Escargot or Worniu. Dev. Biol. 269: 411-420.

Huang, B., Gudi, R., Wu, P., Harris, R.A., Hamilton, J., and Popov, K.M. 1998. Isoenzymes of pyruvate dehydrogenase phosphatase. DNA-derived amino acid sequences, expression, and regulation. J. Biol. Chem. 273: 17680-17688.

Inman, G.J., Nicolas, F.J., and Hill, C.S. 2002. Nucleocytoplasmic shuttling of Smads 2, 3, and 4 permits sensing of TGF- $\beta$ receptor activity. Mol. Cell 10: 283-294.

Kirkpatrick, H., Johnson, K., and Laughon, A. 2001. Repression of dpp targets by binding of brinker to mad sites. J. Biol. Chem. 276: 1821618222 .

Kretzschmar, M., Liu, F., Hata, A., Doody, J., and Massagué, J. 1997. The TGF- $\beta$ mediator Smad1 is directly phosphorylated and functionally activated by the BMP receptor kinase. Genes \& Dev. 11: 984-995.

Kusuda, K., Kobayashi, T., Ikeda, S., Ohnishi, M., Chida, N., Yanagawa, Y., Shineha, R., Nishihira, T., Satomi, S., Hiraga, A., et al. 1998. Mutational analysis of the domain structure of mouse protein phosphatase 2C $\beta$. Biochem. J. 332: 243-250.

Lagna, G., Hata, A., Hemmati-Brivanlou, A., and Massagué, J. 1996. Partnership between DPC4 and SMAD proteins in TGF $\beta$ signalling pathways. Nature 383: 832-836.

Lawson, J.E., Niu, X.D., Browning, K.S., Trong, H.L., Yan, J., and Reed, L.J. 1993. Molecular cloning and expression of the catalytic subunit of bovine pyruvate dehydrogenase phosphatase and sequence similarity with protein phosphatase 2C. Biochemistry 32: 8987-8993.

Livak, K.J. and Schmittgen, T.D. 2001. Analysis of relative gene expression data using real-time quantitative PCR and the $2-\Delta \Delta^{\mathrm{C}_{\mathrm{T}}}$ method. Methods 25: 402-408.

Lo, R.S. and Massagué, J. 1999. Ubiquitin-dependent degradation of TGF$\beta$-activated Smad2. Nat. Cell Biol. 1: 472-478.

Lum, L., Yao, S., Mozer, B., Rovescalli, A., Von Kessler, D., Nirenberg, M., and Beachy, P.A. 2003. Identification of Hedgehog pathway components by RNAi in Drosophila cultured cells. Science 299: 20392045.

Macias-Silva, M., Abdollah, S., Hoodless, P.A., Pirone, R., Attisano, L., and Wrana, J.L. 1996. MADR2 is a substrate of the TGF $\beta$ receptor and phosphorylation is required for nuclear accumulation and signaling. Cell 87: 1215-1224.

Massagué, J., Blain, S.W., and Lo, R.S. 2000. TGF $\beta$ signaling in growth control, cancer, and heritable disorders. Cell 103: 295-309.

Nishihara, A., Fujii, M., Sampath, T.K., Miyazono, K., and Reddi, A.H. 2003. Bone morphogenetic protein signaling in articular chondrocyte differentiation. Biochem. Biophys. Res. Commun. 301: 617-622.

Persson, U., Izumi, H., Souchelnytskyi, S., Itoh, S., Grimsby, S., Engstrom, U., Heldin, C.H., Funa, K., and ten Dijke, P. 1998. The L45 loop in type I receptors for TGF- $\beta$ family members is a critical determinant in specifying Smad isoform activation. FEBS Lett. 434: 83-87.

Raftery, L.A. and Sutherland, D.J. 1999. TGF- $\beta$ family signal transduction in Drosophila development: From Mad to Smads. Dev. Biol. 210: 251-268.

Raftery, L.A., Twombly, V., Wharton, K., and Gelbart, W.M. 1995. Genetic screens to identify elements of the decapentaplegic signaling pathway in Drosophila. Genetics 139: 241-254.

Sekelsky, J.J., Newfeld, S.J., Raftery, L.A., Chartoff, E.H., and Gelbart, W.M. 1995. Genetic characterization and cloning of Mothers against $d p p$, a gene required for decapentaplegic function in Drosophila melanogaster. Genetics 139: 1347-1358.
Shi, Y. and Massagué, J. 2003. Mechanisms of TGF- $\beta$ signaling from cell membrane to the nucleus. Cell 113: 685-700.

Simonot, C., Lerme, F., Louisot, P., and Gateau-Roesch, O. 1997. Submitochondrial localization of the catalytic subunit of pyruvate dehydrogenase phosphatase. FEBS Lett. 401: 158-162.

Souchelnytskyi, S., Tamaki, K., Engstrom, U., Wernstedt, C., ten Dijke, P., and Heldin, C.H. 1997. Phosphorylation of Ser465 and Ser467 in the C terminus of Smad2 mediates interaction with Smad4 and is required for transforming growth factor- $\beta$ signaling. J. Biol. Chem. 272: $28107-28115$.

Sutherland, D.J., Li, M., Liu, X.Q., Stefancsik, R., and Raftery, L.A. 2003. Stepwise formation of a SMAD activity gradient during dorsal-ventral patterning of the Drosophila embryo. Development 130: 57055716.

Tanimoto, H., Itoh, S., ten Dijke, P., and Tabata, T. 2000. Hedgehog creates a gradient of DPP activity in Drosophila wing imaginal discs. Mol. Cell 5: 59-71.

ten Dijke, P. and Hill, C.S. 2004. New insights into TGF- $\beta$-Smad signalling. Trends Biochem. Sci. 29: 265-273.

Thibault, S.T., Singer, M.A., Miyazaki, W.Y., Milash, B., Dompe, N.A., Singh, C.M., Buchholz, R., Demsky, M., Fawcett, R., Francis-Lang, H.L., et al. 2004. A complementary transposon tool kit for Drosophila melanogaster using P and piggyBac. Nat. Genet. 36: 283-287.

Whitman, M. 1998. Smads and early developmental signaling by the TGFß superfamily. Genes \& Dev. 12: 2445-2462.

Xu, L., Kang, Y., Col, S., and Massagué, J. 2002. Smad2 nucleocytoplasmic shuttling by nucleoporins CAN/Nup214 and Nup153 feeds TGF $\beta$ signaling complexes in the cytoplasm and nucleus. Mol. Cell 10: $271-282$ 


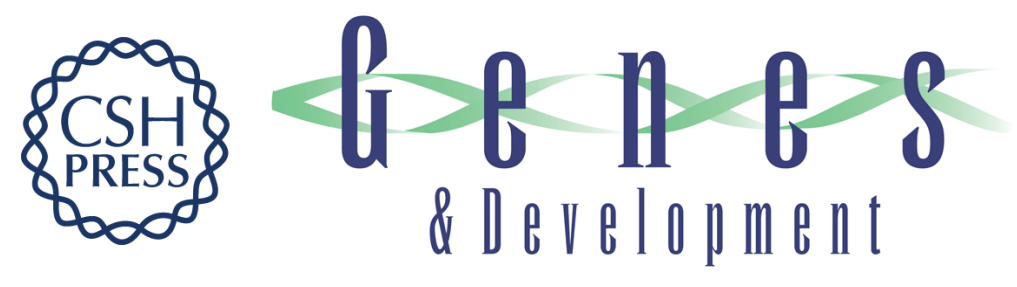

\section{Identification of phosphatases for Smad in the BMP/DPP pathway}

Hong B. Chen, Jiali Shen, Y. Tony Ip, et al.

Genes Dev. 2006, 20:

Access the most recent version at doi:10.1101/gad.1384706

References This article cites 34 articles, 13 of which can be accessed free at: http://genesdev.cshlp.org/content/20/6/648.full.html\#ref-list-1

License

Email Alerting Receive free email alerts when new articles cite this article - sign up in the box at the top Service right corner of the article or click here.

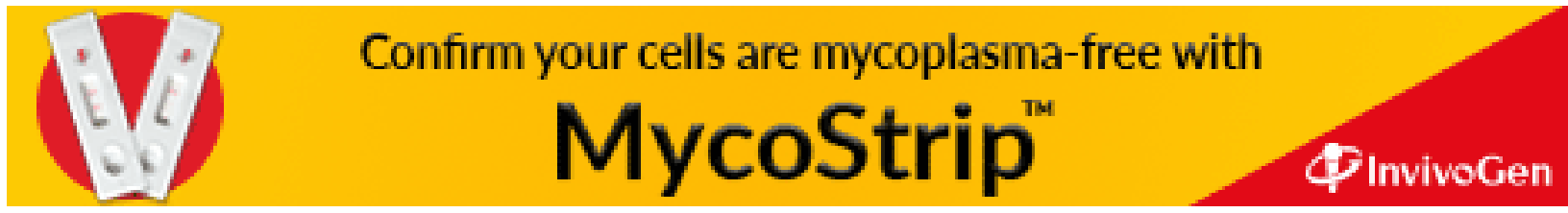

